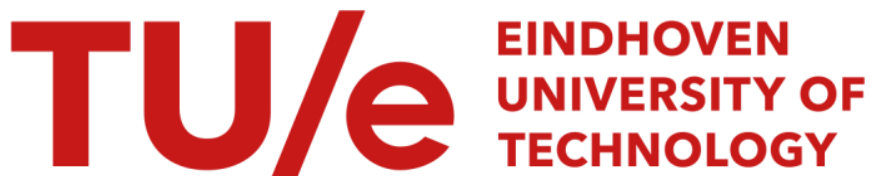

\section{Single, double, and triple quantum dots in Ge/Si nanowires}

Citation for published version (APA):

Froning, F. N. M., Rehmann, M. K., Ridderbos, J., Brauns, M., Zwanenburg, F. A., Li, A., Bakkers, E. P. A. M., Zumbühl, D. M., \& Braakman, F. R. (2018). Single, double, and triple quantum dots in Ge/Si nanowires. Applied Physics Letters, 113(7), [073102]. https://doi.org/10.1063/1.5042501

DOI:

$10.1063 / 1.5042501$

Document status and date:

Published: 13/08/2018

\section{Document Version:}

Publisher's PDF, also known as Version of Record (includes final page, issue and volume numbers)

\section{Please check the document version of this publication:}

- A submitted manuscript is the version of the article upon submission and before peer-review. There can be important differences between the submitted version and the official published version of record. People interested in the research are advised to contact the author for the final version of the publication, or visit the $\mathrm{DOI}$ to the publisher's website.

- The final author version and the galley proof are versions of the publication after peer review.

- The final published version features the final layout of the paper including the volume, issue and page numbers.

Link to publication

\section{General rights}

Copyright and moral rights for the publications made accessible in the public portal are retained by the authors and/or other copyright owners and it is a condition of accessing publications that users recognise and abide by the legal requirements associated with these rights.

- Users may download and print one copy of any publication from the public portal for the purpose of private study or research.

- You may not further distribute the material or use it for any profit-making activity or commercial gain

- You may freely distribute the URL identifying the publication in the public portal.

If the publication is distributed under the terms of Article 25fa of the Dutch Copyright Act, indicated by the "Taverne" license above, please follow below link for the End User Agreement:

www.tue.nl/taverne

Take down policy

If you believe that this document breaches copyright please contact us at:

openaccess@tue.nl

providing details and we will investigate your claim. 


\section{Single, double, and triple quantum dots in Ge/Si nanowires}

F. N. M. Froning, M. K. Rehmann, J. Ridderbos, M. Brauns, F. A. Zwanenburg, A. Li, E. P. A. M. Bakkers, D. M. Zumbühl, and F. R. Braakman

Citation: Appl. Phys. Lett. 113, 073102 (2018); doi: 10.1063/1.5042501

View online: https://doi.org/10.1063/1.5042501

View Table of Contents: http://aip.scitation.org/toc/apl/113/7

Published by the American Institute of Physics

\section{Articles you may be interested in}

Hot carrier impact on photovoltage formation in solar cells

Applied Physics Letters 113, 071103 (2018); 10.1063/1.5043155

Direct-to-indirect electronic state transition in dynamically compressed GaAs quantum wells Applied Physics Letters 113, 072101 (2018); 10.1063/1.5038723

Enhancing the sensitivity of a single electron spin sensor by multi-frequency control Applied Physics Letters 113, 072401 (2018); 10.1063/1.5042796

Transport properties of a few nanometer-thick TiSe 2 films grown by molecular-beam epitaxy Applied Physics Letters 113, 073101 (2018); 10.1063/1.5039493

An ultra-thin coplanar waveguide filter based on the spoof surface plasmon polaritons Applied Physics Letters 113, 071101 (2018); 10.1063/1.5045069

Large Hall angle-driven magneto-transport phenomena in topological Dirac semimetal $\mathrm{Cd}_{3} \mathrm{As}_{2}$ Applied Physics Letters 113, 072104 (2018); 10.1063/1.5037789

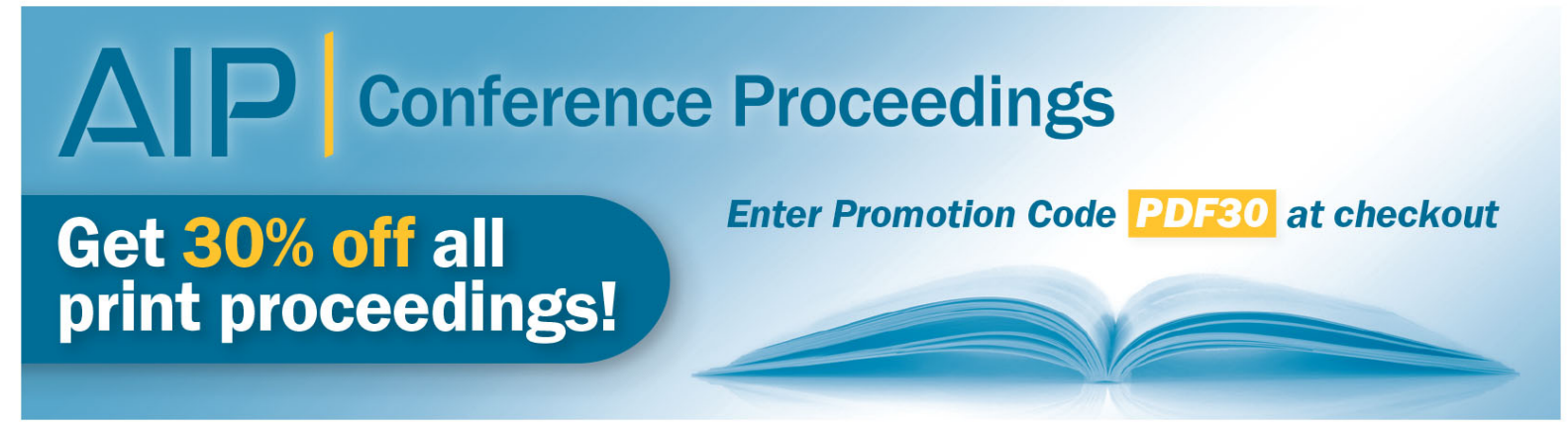




\title{
Single, double, and triple quantum dots in Ge/Si nanowires
}

\author{
F. N. M. Froning, ${ }^{1}$ M. K. Rehmann, ${ }^{1}$ J. Ridderbos, ${ }^{2}$ M. Brauns, ${ }^{2}$ F. A. Zwanenburg, ${ }^{2}$ A. Li, ${ }^{3}$ \\ E. P. A. M. Bakkers, ${ }^{3,4}$ D. M. Zumbühl, ${ }^{1}$ and F. R. Braakman ${ }^{1, a)}$ \\ ${ }^{1}$ Department of Physics, University of Basel, Klingelbergstrasse 82, 4056 Basel, Switzerland \\ ${ }^{2}$ NanoElectronics Group, MESA+ Institute for Nanotechnology, University of Twente, P.O. Box 217, \\ 7500 AE Enschede, The Netherlands \\ ${ }^{3}$ Department of Applied Physics, Eindhoven University of Technology, P.O. Box 513, 5600 MB Eindhoven, \\ The Netherlands \\ ${ }^{4}$ QuTech and Kavli Institute of Nanoscience, Delft University of Technology, 2600 GA Delft, The Netherlands
}

(Received 1 June 2018; accepted 31 July 2018; published online 15 August 2018)

\begin{abstract}
We report highly tunable control of holes in $\mathrm{Ge} / \mathrm{Si}$ core/shell nanowires. We demonstrate the ability to create single quantum dots of various sizes, with low hole occupation numbers and clearly observable excited states. For the smallest dot size, we observe indications of single-hole occupation. Moreover, we create double and triple tunnel-coupled quantum dot arrays. In the double quantum dot configuration, we observe Pauli spin blockade. These results open the way to perform hole spin qubit experiments in these devices. Published by AIP Publishing.

https://doi.org/10.1063/1.5042501
\end{abstract}

Single hole spins confined in quantum dots (QDs) in Ge/ $\mathrm{Si}$ core/shell nanowires (NWs) combine several advantageous properties which make them potentially very powerful quantum bits. ${ }^{1,2}$ The natural abundance of non-zero nuclear spins in both silicon and germanium is relatively small and can be further reduced to a negligible amount by isotopic purification. Furthermore, hole spins have no contact hyperfine interaction due to their p-type wavefunction. These properties make hole spin qubits in silicon and germanium resilient against dephasing via interaction with nuclear spins.

A particularly promising feature of hole spins in $\mathrm{Ge} / \mathrm{Si}$ core/shell NWs is the nature of spin-orbit interaction (SOI) in this system. Confinement to one dimension gives rise to an effective SOI in the valence band, which is predicted to be both strong and tunable, ${ }^{3,4}$ enabling fast all-electrical spin manipulation. An external electric field can be used to set the strength of this SOI. This promises the capability of electrical gating of the SOI, allowing to switch to a large SOI for high interaction strengths and fast quantum operations, or to turn off SOI for increased qubit coherence. Furthermore, this SOI results in a Landé g-factor that is locally tunable by external electric as well as magnetic fields. ${ }^{5,6}$ Local control over the g-factor makes it possible to selectively address individual spin qubits and allows for selective coupling to microwave cavities. ${ }^{7}$

The confinement of single holes in QDs is an important step towards implementation of the basic ingredients of experimental quantum computation using hole spin qubits. ${ }^{1}$ Single QDs form the fundamental building blocks, and it is therefore imperative to be able to reliably form and characterize them. ${ }^{8}$ Moreover, a high level of control over the exact position and shape of individual QDs is required to accurately tune level splittings, ${ }^{3}$ spin relaxation times,,${ }^{9,10}$ and tunnel coupling strengths.

In addition to single QDs, tunnel-coupled double QDs are of particular interest, since these are platforms for spin-

\footnotetext{
${ }^{\text {a) }}$ Author to whom correspondence should be addressed: floris.braakman@ unibas.ch
}

to-charge conversion schemes facilitating spin read-out and coupling of spins to microwave cavities. ${ }^{11-13}$ Spin states of double and triple QDs can be used as qubit encodings which are insensitive to fluctuations of a uniform magnetic field or of magnetic field gradients. ${ }^{14,15}$ Moreover, quantum operations on these qubits may be performed using different mechanisms than for single spin qubits, for instance, only relying on the Heisenberg exchange interaction. ${ }^{16,17}$ Finally, double as well as triple QDs feature charge states with an increased dipole moment, potentially leading to enhanced coupling strengths of spin qubits to microwave cavities. ${ }^{13}$

In this letter, we demonstrate a large amount of control over the formation of single, double, and triple QDs in $\mathrm{Ge} / \mathrm{Si}$ NWs, all with a low hole occupation number. Using five bottom gate electrodes, we tune the size and position of single QDs defined in the NW. Furthermore, we form tunnelcoupled double and triple QDs. In the double QD configuration, we observe Pauli spin blockade ${ }^{9,18}$ (PSB).

We use a $\mathrm{Ge} / \mathrm{Si} \mathrm{NW}^{19}$ with an estimated Ge core radius of $10 \mathrm{~nm}$ and Si shell thickness of $2.5 \mathrm{~nm}$ (see Fig. 1). Five $\mathrm{Ti} / \mathrm{Pd}$ bottom gate electrodes are lithographically defined on a p++-doped Si substrate covered with $290 \mathrm{~nm}$ thermal oxide. The bottom gates have a thickness of $\sim 15 \mathrm{~nm}$, a width of $20 \mathrm{~nm}$, and are equally spaced with a pitch of $50 \mathrm{~nm}$. On either side of these gates, a plateau gate [green in Fig. 1(b)] is defined, which serves to prevent bending of the NW. The bottom gates are subsequently covered by a layer of $\mathrm{Al}_{2} \mathrm{O}_{3}$ of thickness $20 \mathrm{~nm}$ through atomic layer deposition at $225^{\circ} \mathrm{C}$. In a next step, the NW is placed deterministically on top of the bottom gates using a micromanipulator setup. Electrical contact to the $\mathrm{NW}$ is made through two Ti/Pd $(\sim 0.5 / 60 \mathrm{~nm})$ contact pads, which are lithographically defined and metallized after a brief HF dip to strip the NWs native oxide.

Due to the type-II staggered band alignment of silicon and germanium, a hole gas accumulates in the core. ${ }^{20} \mathrm{By}$ applying positive voltages to the gate electrodes, the hole density can be depleted locally, resulting in the formation of 
(a)

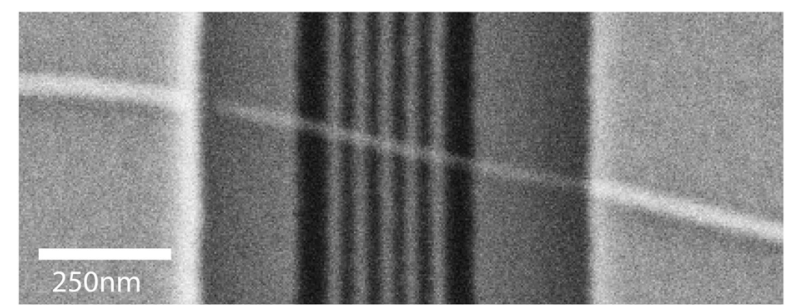

(b)

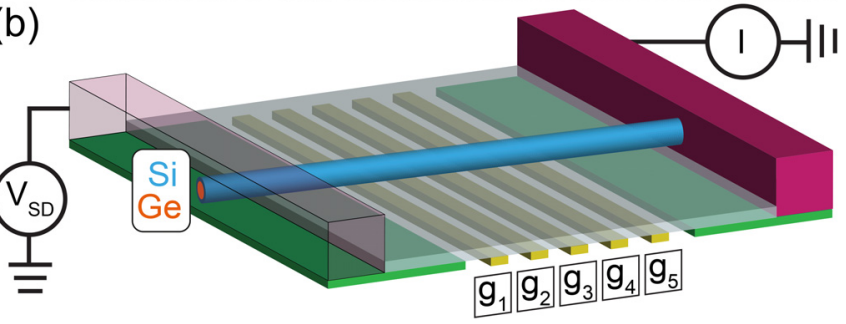

FIG. 1. (a) Scanning electron micrograph of a device similar to the one used in this work. (b) Schematic overview of device and measurement setup. The NW is shown in blue, with the core in orange, bottom gates are in yellow and green, and contacts in purple.

QDs. We perform transport measurements by applying a dc source-drain bias $V_{S D}$ over the NW and measuring the differential conductance using standard lock-in techniques with a small ac excitation in the range of $20-100 \mu \mathrm{V}$ applied to the source contact. All measurements were performed at a temperature of $1.4 \mathrm{~K}$, without application of an external magnetic field, and with the doped part of the substrate grounded.

Figure 2(a) gives an overview of the different configurations of biased gates and dot sizes that were studied. QDs can be formed using two, three, four, or five neighboring gates. For each dot size, the outer two gates [red in Fig. 2(a)] form tunnel barriers between the QD and the source and drain reservoirs. The voltage on individual or multiple middle gates [green in Fig. 2(a)] are used to tune the electrochemical potential of the QD. Unused gates [white in Fig. 2(a)] are grounded. In Figs. 2(b) and 2(c), measured charge stability diagrams (Coulomb diamonds) are shown for the case of a single QD formed by two and three neighboring gates, respectively [see Fig. 2(a), top panels]. Similar measurements were made for larger QDs formed by four and five gates. In case of the QD defined by two adjacent gates, we find that sweeping the voltage on these gates has a large effect on the tunnel barriers defining the dot. As a result, only a few charge transitions can be observed for this configuration. For the other dot sizes, the tunnel barriers are much less affected by the voltage on one of the middle gates, and we observe a large number of regular Coulomb diamonds.

Table I summarizes parameters extracted from the Coulomb diamond measurements. In Fig. 3, values of the hole addition energy $E_{\text {add }}$ are plotted, which were extracted from the height of the Coulomb diamonds. We find that $E_{\text {add }}$ is the largest for the smallest dot and decreases for increasing dot size, in agreement with the expectation that both charging energy and orbital level splittings decrease with the dot size.

The conductance measurements feature additional resonances at higher values of $V_{S D}{ }^{22}$ We extract energies for these resonances by averaging the difference of the first (a)
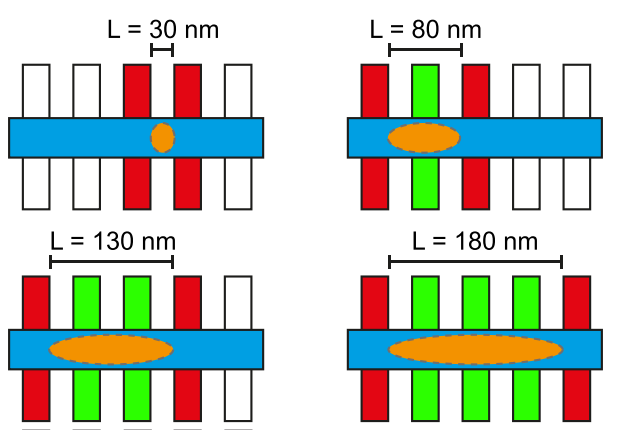

$\begin{array}{lllll}g_{1} & g_{2} & g_{3} & g_{4} & g_{5}\end{array}$

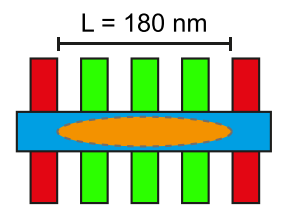

(b)

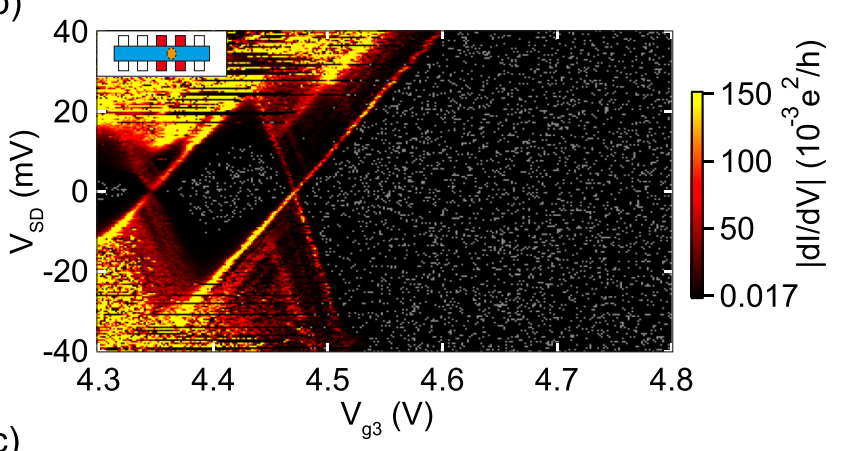

(c)

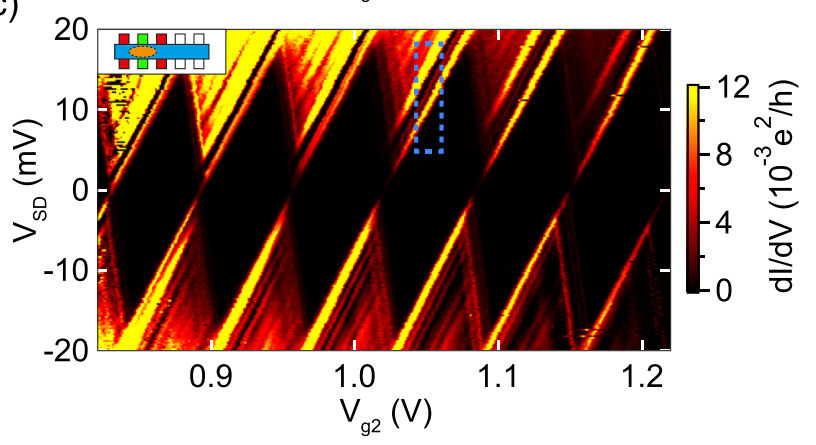

FIG. 2. (a) Schematic picture of the gate configurations used to form QDs (orange) of different lengths using 2, 3, 4, and 5 gates, respectively. (b) Lock-in signal $d I / d V$ versus $V_{S D}$ and $V_{g 3}$ of QD formed by two gates. To enhance contrast, values below the colorscale were given a grey color. Here, $V_{g 4}=1700 \mathrm{mV}$. (c) Lock-in signal $d I / d V$ versus $V_{S D}$ and $V_{g 2}$ of QD formed by three gates. Here, $V_{g 1}=2000 \mathrm{mV}$ and $V_{g 3}=4000 \mathrm{mV}$. Blue dashed rectangle shows an example of an averaging window used to extract excited state energies. Insets in (b) and (c) schematically show used gate configurations.

resonance and the ground state transition, in windows similar to the one drawn in Fig. 2(c). Here, we convert the difference in $V_{S D}$ to energy using lever arms determined from the slopes of each Coulomb diamond. The third column of Table I lists typical energies $E_{\text {orb }}$ found in this way for the different dot sizes. Consistent with the level splitting of orbital hole states ${ }^{21,25} E_{\text {orb }}$ depends strongly on the longitudinal dot size, with smaller dots featuring higher values of $E_{\text {orb }}$. Note that

TABLE I. Typical extracted single dot parameters: addition energies $E_{\text {add }}$, excited state energies $E_{\text {orb }}$, lithographically defined distances $L$ between gates creating QD tunnel barriers, and estimated hole numbers $N_{\text {est }}$.

\begin{tabular}{lcccc}
\hline \hline No. of gates & $E_{\text {add }}(\mathrm{meV})$ & $E_{\text {orb }}(\mathrm{meV})$ & $L(\mathrm{~nm})$ & $N_{\text {est }}$ \\
\hline 2 & 26 & 12.8 & 30 & 1 , see main text \\
3 & 17 & 4.8 & 80 & 15 \\
4 & 13 & 2.1 & 130 & 35 \\
5 & 10 & 1.3 & 180 & 38 \\
\hline \hline
\end{tabular}




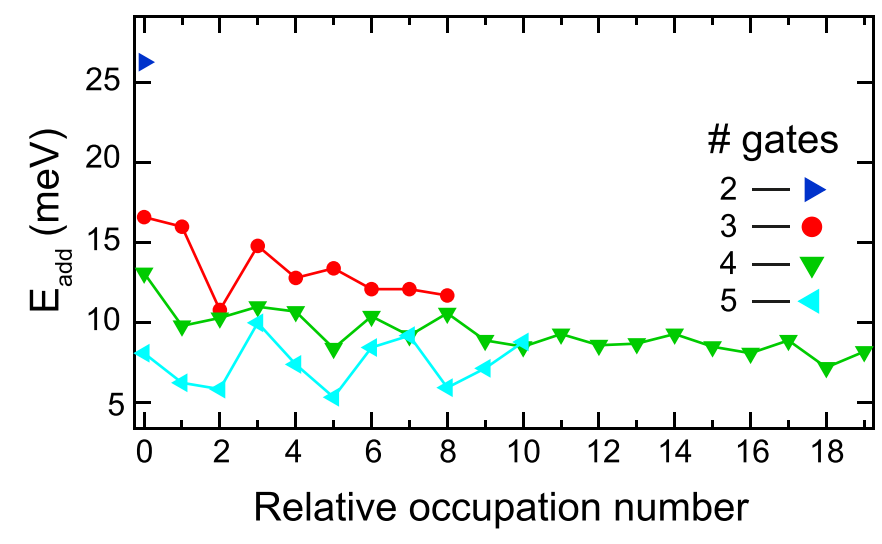

FIG. 3. Extracted values of $E_{\text {add }}$ for various QD lengths as a function of relative occupation number.

incomplete knowledge of the exact confinement potential and the hole effective mass makes it difficult to compare our measurements to a theoretical model of orbital level splitting. Furthermore, we estimate the lowest measurable hole occupation number $N_{\text {est }}$ for the different dot sizes by comparing the used gate voltages with pinch-off voltages obtained at high $V_{S D}$. For dots formed by 3-5 neighboring gates, we find relatively low occupation numbers ranging from 15 to 38 (see Table I). This method is not reliable for QDs defined by only two gates, since both gates directly define the tunnel barriers of the dot. However, several indications suggest that single-hole occupation is reached in this case. First of all, the last Coulomb diamond edge visible in Fig. 2(b) increases linearly up to at least $\left|V_{S D}\right|=40 \mathrm{mV}$. Furthermore, even at high $V_{S D}$, no features involving tunneling of multiple holes are observed for the last visible Coulomb diamond (which would appear as lines intersecting the diamond edges on the high gate voltage side). We do find multiple resonances in the last diamond for low $V_{S D}$, which could arise from tunneling involving excited states. However, the splitting of these lines is lower than that found for the larger dots. Therefore, it is unlikely that these resonances correspond to excited orbital states in a small QD. Furthermore, we observe (not shown) that the splitting of the resonances strongly depends on gate voltages applied to $g_{2}$ and $g_{5}$ (flanking the barriers of the dot), again making it implausible that they correspond to excited orbital states. ${ }^{23}$ A likely explanation is that these lines arise from modulation of the reservoir density of states. $^{23-25}$ Finally, the energy of the first excited state in the second Coulomb diamond in Fig. 2(b) (around $V_{g 3}=4.35 \mathrm{~V}$ ) appears to be significantly reduced with respect to that found in the last diamond, consistent with an exchange energy appearing for two-hole states. More conclusive evidence of single hole occupation could be obtained by using a charge sensor. $^{26}$
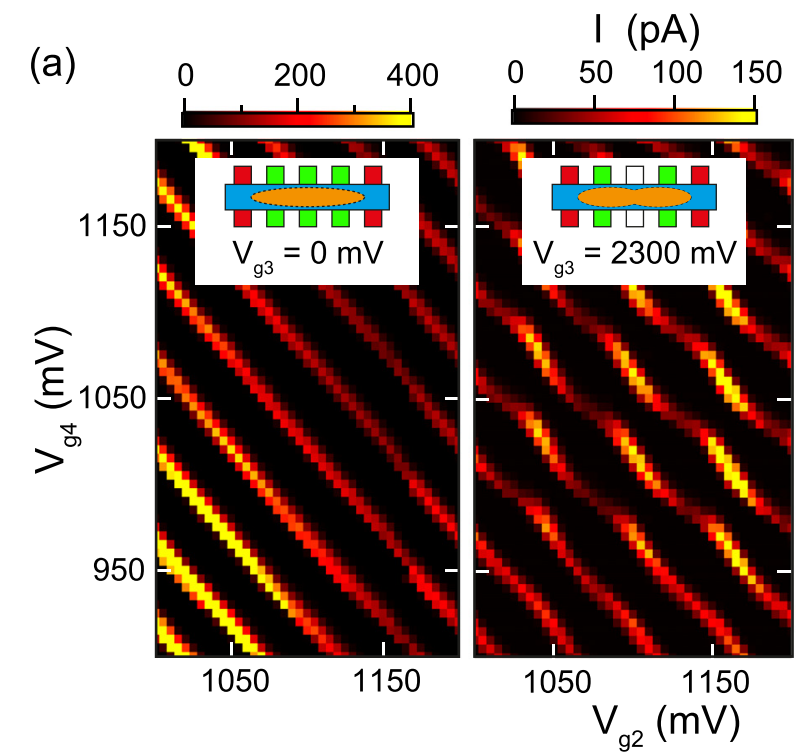

(b)

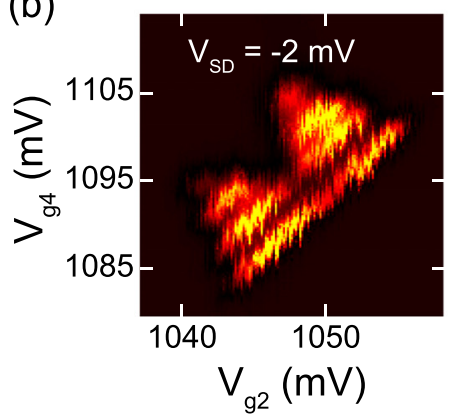

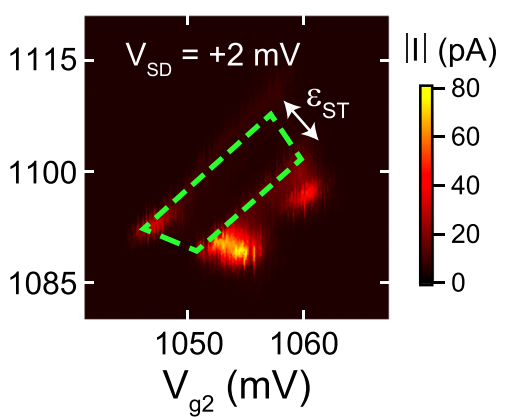
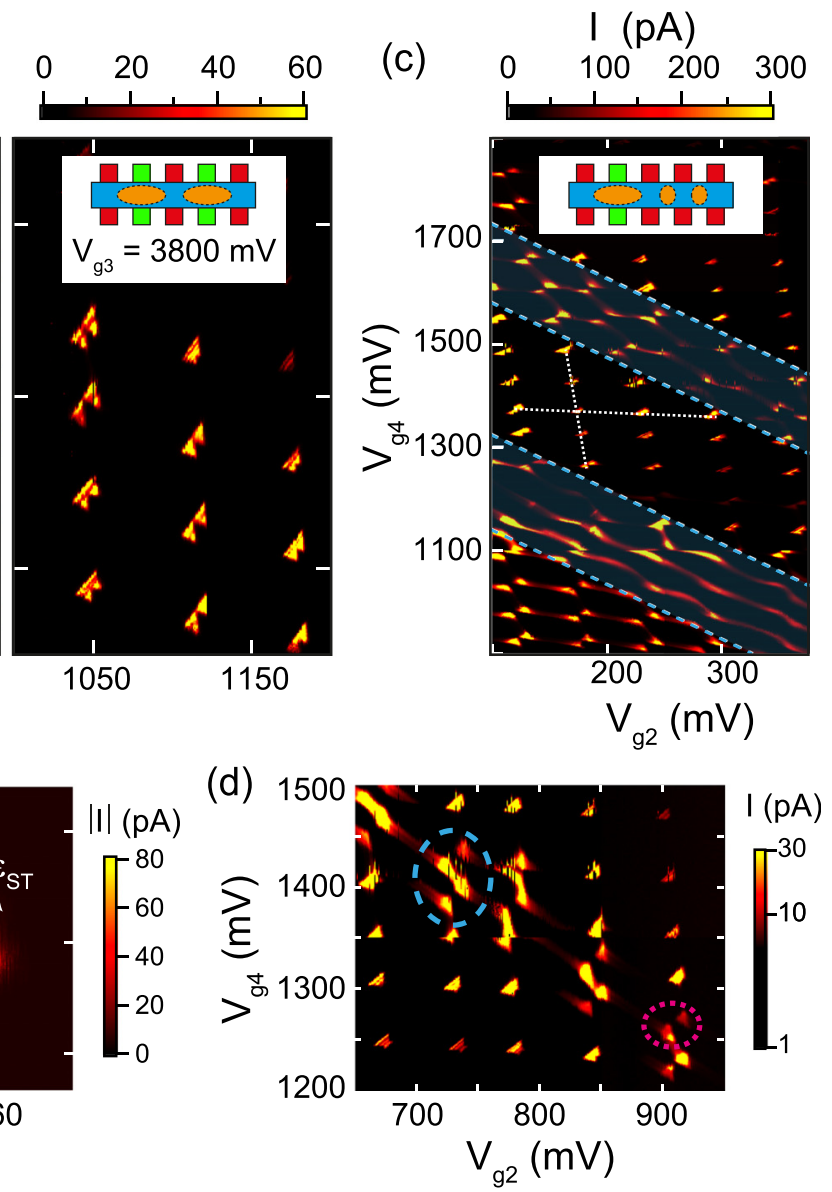

FIG. 4. (a) Charge stability diagrams for different values of the voltage on $g_{3}$, showing a transition from a single QD to a double QD, at $V_{S D}=2 \mathrm{mV}$. Insets schematically show QD configurations. (b) Zoom-in of a pair of bias triangles, at $V_{g 3}=3800 \mathrm{mV}$. Plotted is the dc current for positive and negative $V_{S D}$. The strong reduction in the area enclosed by the dashed green line indicates the presence of Pauli spin blockade. (c) Charge stability diagram with highlighted (shaded blue regions) triple QD features. White dotted lines indicate the slope of charge transitions of the outer two dots. (d) Charge stability diagram of triple QD. Dashed blue and dotted pink circles highlight triple dot resonances. In (c) and (d), $V_{g 1}=2000 \mathrm{mV}, V_{g 3}=3800 \mathrm{mV}$, and $V_{g 5}=2800 \mathrm{mV}$. 
Finally, we observed in multiple devices that QDs formed by three or more gates tend to split up when biasing the center gates too positively. This impedes reaching singlehole occupation for the larger dot sizes. Moreover, conductance becomes too low to measure when increasing the gate voltages, again potentially preventing the observation of single-hole occupation regimes. In the device studied here, this seems to be not the case for the dot made by two gates. The conductance exceeds $0.1 e^{2} / h$ on the last diamond in Fig. 2(b), thus adding more evidence for the single-hole regime.

Next, we demonstrate controllable formation of double QDs. As shown in the charge stability diagrams in Fig. 4(a), a single QD formed by five gates can be continuously split up into a double tunnel-coupled QD, by increasing the voltage on gate $g_{3}$. Here, the voltage on gates $g_{2}$ and $g_{4}$ are swept and the current through the NW is measured for each point. The leftmost charge stability diagram shows single-dot behavior, in which diagonal lines are Coulomb peaks corresponding to sequential addition of single holes to the dot. The middle panel shows a charge stability diagram of a double QD featuring high coupling between the dots, as evidenced by the bending of the charging lines. The right panel shows conductance only when the electrochemical potentials of the two dots are aligned, in the form of bias triangles. ${ }^{27}$ The absence of conductance along the charging lines indicates that significant cotunneling with the lead reservoirs can be avoided. These measurements indicate that we have a large amount of control over the capacitive coupling and tunnel-coupling between the two QDs.

Pauli spin blockade is a basic ingredient of many spin qubit experiments, in which interdot transitions are blocked for spin triplet but not for singlet states. ${ }^{9,18}$ As such, it forms a means of reading out spin qubit states. When measuring the conductance through a double QD, the blockade may be observed for one sign of $V_{S D}$, but not for the other. In this work, the relevant spin states are those of Kramers doublets formed by mixed heavy hole and light hole states. ${ }^{3}$

We observe signatures of PSB at several interdot transitions when measuring bias triangles for positive and negative $V_{S D}$, in the form of a region of reduced conductance inside the bias triangles for one sign of $V_{S D}$. Figure 4(b) focuses on one such an interdot transition where the current inside the region indicated by the dashed green line is suppressed by roughly a factor 10 for positive $V_{S D}$. The size of the blockaded region is determined by the singlet-triplet splitting $\epsilon_{S T}$ in the single dots [see white arrow in Fig. 4(b), right panel]. We find $\varepsilon_{S T}$ to be $1 \mathrm{meV}$, which compares well with other measurements. ${ }^{28,29}$ Moreover, we observe a leakage current that depends on the detuning of the electrochemical potentials in the two dots and on the magnitude of an applied magnetic field. In particular, we find a small leakage current at low detuning that is consistent with an effective $\mathrm{T}(1,1)$ to $\mathrm{S}(0,2)$ transition, and a larger current at higher detuning consistent with a $\mathrm{T}(1,1)$ to $\mathrm{T}(0,2)$ transition. Various processes may lift PSB, including spin-flip cotunneling, spin-flip reservoir exchange, ${ }^{30}$ hyperfine interaction, and SOI. ${ }^{28,29,31}$ The resulting leakage current thus forms a probe to detect the strength of these processes, but a detailed study of this goes beyond the scope of the present work.
We find that the double QD can be further subdivided into a triple QD, by increasing the voltage on $g_{4}$. In this case, the triple dot is likely composed of two small QDs between gate pairs $g_{3}-g_{4}$ and $g_{4}-g_{5}$, as well as a larger QD between $g_{1}-g_{3}$. In the charge stability diagram shown in Fig. 4(c), triple dot features appear as lines with enhanced conductance with an intermediate slope (see dashed blue lines). Figure 4(d) shows a zoomed-in region of the triple QD charge stability diagram. Similar to bias triangles in a double QD, conductance is enhanced when the electrochemical potential of the center dot is aligned with that of one of the outer dots [dotted pink circle in Fig. 4(d)], or when the electrochemical potentials of all three dots are aligned [dashed blue circle in Fig. 4(d)]. ${ }^{32,33}$ The fact that we also observe conductance at points corresponding to QD bias triangles suggests that there is cotunneling involving the center dot present in the measurements, resulting in conductance even when only the electrochemical potentials of two out of three dots are aligned.

The demonstration of tunable single, double, and triple QDs opens the way to perform spin qubit experiments with few holes in these devices. Reaching the single-hole regime is particularly important, as it makes single and two-qubit operations much more straightforward. Overall, we observe very good repeatability of the measurements, with gate voltage changes of $1 \mathrm{~V}$ leading to no observable shifts in charge stability diagrams. These results enable several follow-up experiments. In particular, the strength and electric field dependence of the SOI could be determined from magnetic field dependence of leakage current in a double QD in the PSB regime. ${ }^{28,29,31}$ Moreover, we expect that a slightly different gate design than used here will enable reaching singlehole occupation in a controllable way.

We thank C. Kloeffel, D. Loss, and M. Rancic for helpful discussions. We acknowledge the support of the Swiss National Science Foundation (Ambizione Grant No. PZOOP2161284/1, Project Grant No. 157213, and Project Grant No. 179024), the Swiss Nanoscience Institute, the European Microkelvin Platform EMP, the NCCR Quantum Science and Technology (QSIT), and the Netherlands Organization for Scientific Research (NWO).

${ }^{1}$ D. Loss and D. P. DiVincenzo, Phys. Rev. A 57, 120 (1998).

${ }^{2}$ C. Kloeffel and D. Loss, Annu. Rev. Condens. Matter Phys. 4, 51-81 (2013).

${ }^{3}$ C. Kloeffel, M. Trif, and D. Loss, Phys. Rev. B 84, 195314 (2011).

${ }^{4}$ C. Kloeffel, M. J. Rancic, and D. Loss, Phys. Rev. B 97, 235422 (2018).

${ }^{5}$ F. Maier, C. Kloeffel, and D. Loss, Phys. Rev. B 87, 161305 (2013).

${ }^{6}$ M. Brauns, J. Ridderbos, A. Li, E. P. A. M. Bakkers, and F. A. Zwanenburg, Phys. Rev. B 93, 121408 (2016).

${ }^{7}$ C. Kloeffel, M. Trif, P. Stano, and D. Loss, Phys. Rev. B 88, 241405(R) (2013).

${ }^{8}$ M. Brauns, J. Ridderbos, A. Li, E. P. A. M. Bakkers, W. G. van der Wiel, and F. A. Zwanenburg, Appl. Phys. Lett. 109, 143113 (2016).

${ }^{9}$ R. Hanson, L. P. Kouwenhoven, J. R. Petta, S. Tarucha, and L. M. K. Vandersypen, Rev. Mod. Phys. 79, 1217-1265 (2007).

${ }^{10}$ L. C. Camenzind, L. Yu, P. Stano, J. Zimmermann, A. C. Gossard, D. Loss, and D. M. Zumbühl, "Hyperfine-phonon spin relaxation in a singleelectron GaAs quantum dot," Nat. Comm. (to be published), e-print arXiv:1711.01474.

${ }^{11}$ N. Samkharadze, G. Zheng, N. Kalhor, D. Brousse, A. Sammak, U. C. Mendes, A. Blais, G. Scappucci, and L. M. K. Vandersypen, Science 359, 1123-1127 (2018). 
${ }^{12}$ X. Mi, M. Benito, S. Putz, D. M. Zajac, J. M. Taylor, G. Burkard, and J. R. Petta, Nature 555, 599-603 (2018).

${ }^{13}$ A. J. Landig, J. V. Koski, P. Scarlino, U. C. Mendes, A. Blais, C. Reichl, W. Wegscheider, A. Wallraff, K. Ensslin, and T. Ihn, "Coherent spin-qubit photon coupling," Nature (to be published), e-print arXiv:1711.01932.

${ }^{14}$ J. M. Taylor, H.-A. Engel, W. Dür, A. Yacoby, C. M. Marcus, P. Zoller, and M. D. Lukin, Nat. Phys. 1, 177-183 (2005).

${ }^{15}$ J. M. Taylor, V. Srinivasa, and J. Medford, Phys. Rev. Lett. 111, 050502 (2013).

${ }^{16}$ D. P. DiVincenzo, D. Bacon, J. Kempe, G. Burkard, and K. B. Whaley, Nature 408, 339-342 (2000).

${ }^{17}$ J. Medford, J. Beil, J. M. Taylor, S. D. Bartlett, A. C. Doherty, E. I. Rashba, D. P. DiVincenzo, H. Lu, A. C. Gossard, and C. M. Marcus, Nat. Nanotechnol. 8, 654-659 (2013).

${ }^{18}$ K. Ono, D. G. Austing, Y. Tokura, and S. Tarucha, Science 297, 1313-1317 (2002).

${ }^{19}$ S. Conesa-Boj, A. Li, S. Koeling, M. Brauns, J. Ridderbos, T. T. Nguyen, M. A. Verheijen, P. M. Koenraad, F. A. Zwanenburg, and E. P. A. M. Bakkers, Nano Lett. 17, 2259-2264 (2017).

${ }^{20}$ W. Lu, J. Xiang, B. P. Timko, Y. Wu, and C. M. Lieber, Proc. Natl. Acad. Sci. U.S.A. 102, 10046-10051 (2005).

${ }^{21}$ L. P. Kouwenhoven, D. G. Austing, and S. Tarucha, Rep. Prog. Phys. 64, 701-736 (2001).

${ }^{22}$ S. Roddaro, A. Fuhrer, P. Brusheim, C. Fasth, H. Q. Xu, L. Samuelson, J. Xiang, and C. M. Lieber, Phys. Rev. Lett. 101, 186802 (2008).
${ }^{23}$ M. Möttönen, K. Y. Tan, K. W. Chan, F. A. Zwanenburg, W. H. Lim, C. C. Escott, J.-M. Pirkkalainen, A. Morello, C. Yang, J. A. van Donkelaar, A. D. C. Alves, D. N. Jamieson, L. C. L. Hollenberg, and A. S. Dzurak, Phys. Rev. B 81, 161304 (2010).

${ }^{24}$ M. T. Björk, C. Thelander, A. E. Hansen, L. E. Jensen, M. W. Larsson, L. R. Wallenberg, and L. Samuelson, Nano Lett. 4, 1621-1625 (2004).

${ }^{25}$ C. C. Escott, F. A. Zwanenburg, and A. Morello, Nanotechnology 21, 274018 (2010).

${ }^{26}$ Y. Hu, H. O. H. Churchill, D. J. Reilly, J. Xiang, C. M. Lieber, and C. M. Marcus, Nat. Nanotechnol. 2, 622-625 (2007).

${ }^{27}$ W. G. van der Wiel, S. De Franceschi, J. M. Elzerman, T. Fujisawa, S. Tarucha, and L. P. Kouwenhoven, Rev. Mod. Phys. 75, 1-22 (2002).

${ }^{28}$ M. Brauns, J. Ridderbos, A. Li, E. P. A. M. Bakkers, W. G. van der Wiel, and F. A. Zwanenburg, Phys. Rev. B 94, 041411(R) (2016).

${ }^{29}$ A. Zarassi, Z. Su, J. Danon, J. Schwenderling, M. Hocevar, B. M. Nguyen, J. Yoo, S. A. Dayeh, and S. M. Frolov, Phys. Rev. B 95, 155416 (2017).

${ }^{30}$ D. E. F. Biesinger, C. P. Scheller, B. Braunecker, J. Zimmerman, A. C. Gossard, and D. M. Zumbühl, Phys. Rev. Lett. 115, 106804 (2015).

${ }^{31}$ R. Li, F. E. Hudson, A. S. Dzurak, and A. R. Hamilton, Nano Lett. 15, 7314-7318 (2015).

${ }^{32}$ D. Schröer, A. D. Greentree, L. Gaudreau, K. Eberl, L. C. L. Hollenberg, J. P. Kotthaus, and S. Ludwig, Phys. Rev. B 76, 075306 (2007).

${ }^{33}$ G. Granger, L. Gaudreau, A. Kam, M. Pioro-Ladrière, S. A. Studenikin, Z. R. Wasilewski, P. Zawadzki, and A. S. Sachrajda, Phys. Rev. B 82, 075304 (2010). 